\title{
Médiévales
}

Langues, Textes, Histoire

67 | automne 2014

Histoires de Bohême

\section{Le Miroir historial de Jacques d'Armagnac : un monument bibliophilique pour un prince ambitieux}

The Miroir historial of Jacques d'Armagnac : a Bibliophilic Monument for an Ambitious Prince

\section{Cécile Ranvier}

\section{OpenEdition}

Journals

Édition électronique

URL : https://journals.openedition.org/medievales/7437

DOI : 10.4000/medievales. 7437

ISSN : $1777-5892$

\section{Éditeur}

Presses universitaires de Vincennes

\section{Édition imprimée}

Date de publication : 31 décembre 2014

Pagination : 143-168

ISBN : 978-2-84292-422-5

ISSN : 0751-2708

\section{Référence électronique}

Cécile Ranvier, «Le Miroir historial de Jacques d'Armagnac : un monument bibliophilique pour un prince ambitieux », Médiévales [En ligne], 67 | automne 2014, mis en ligne le 31 décembre 2016, consulté le 22 avril 2022. URL : http://journals.openedition.org/medievales/7437 ; DOI : https:// doi.org/10.4000/medievales.7437 


\section{Cécile Ranvier}

\section{Le Miroir historial de Jacques d'Armagnac: un monument bibliophilique pour un prince ambitieux}

Parmi les manuscrits du Miroir historial qui nous sont parvenus, la copie exécutée pour Jacques d'Armagnac durant la seconde moitié du $\mathrm{Xv}^{\mathrm{e}}$ siècle impressionne par son luxe ostentatoire, témoignage des moyens exceptionnels déployés pour sa réalisation. Sa reliure à ais de bois, qui protège plus d'un millier de feuillets ornés de près de cinq cents miniatures, constitue l'écrin somptueux de la version française du texte de Vincent de Beauvais. À l'aube de la commande de cette monumentale pièce bibliophilique, en 1459, le duc de Nemours, prince de sang royal et pair de France, est devenu l'un des seigneurs les plus puissants du royaume grâce aux faveurs accordées par Charles VII puis Louis XI, avant le conflit qui l'opposa à ce dernier. Ce fleuron de la collection du duc, témoignage des prémices de son activité bibliophilique en trois fastueux volumes, n'est pourtant utilisé le plus souvent par les chercheurs qu'à titre de référent ponctuel. Il n'a en effet jamais bénéficié jusqu'à présent d'une véritable analyse scientifique $^{1}$. L'examen de l'iconographie d'un petit groupe de miniatures - en l'occurrence les vingt-six images des cycles marial et christique -, confronté aux données historiques, textuelles et codicologiques, permet pourtant de dresser un portrait nuancé de Jacques d'Armagnac - homme politique ambitieux et mécène éclairé. Une telle analyse est également à même d'aiguiser notre connaissance des méthodes de travail des ateliers d'enluminure de la fin du Moyen Âge, à l'exemple de l'atelier parisien du Maître de Rolin et de Maître François, responsables de l'ornementation

1. Susan A.Blackman, auteure d'une thèse consacrée à l'activité bibliophilique du duc, n'en donne que les principales caractéristiques codicologiques: S. A.Blackman, The Manuscripts and Patronage of Jacques d'Armagnac, Duke of Nemours, 1433-1477, Ph. D., Pittsburgh, 1993 (non édité). 
du Miroir historial, dont l'attribution précise fait cependant encore débat aujourd'hui ${ }^{2}$.

\section{Une œuvre exceptionnelle}

La durée de transcription des trois volumes constitutifs du premier manuscrit commandé par Jacques d'Armagnac est attestée par le colophon encore visible à la fin du dernier ouvrage: "fut escript et commancé le p[rése]nt livre par moy Gilles Gracien [en] l'an LIX et fut finy les premier jour de septembre, mil CCCC soixante et trois ». Probablement copié à partir de deux modèles différents achetés par le duc de Nemours à des libraires parisiens sous forme de cahiers non reliés ${ }^{3}$, le texte s'étend sur près de 1352 feuillets. Mesurant 478 sur 330 millimètres, ceux-ci ont été découpés dans un parchemin de peau de veau ou de vachette. Réparti sur deux colonnes de quarante-neuf lignes chacune, le texte transcrit par Gilles Gracien de Poitiers est structuré par des encadrements et des bordures à vignetures et feuilles d'acanthes et décoré de bouts de ligne, d'initiales champies ou fleuries sur fond d'or bruni et de cinq cent trois miniatures. La plupart sont carrées et larges d'une colonne. Placées au-dessus des rubriques inscrites à l'encre rouge, elles sont réparties dans les trois volumes de manière irrégulière. Cependant, chacun des trente-deux livres qui composent le Miroir historial est précédé d'une image rectangulaire large d'une colonne et demie, à l'exception du premier et du dix-septième livres, illustrés d'une miniature dont les dimensions exceptionnelles - plus de la moitié de l'espace d'écriture - mettent en évidence la division textuelle traditionnelle ${ }^{4}$.

L'organisation rigoureuse du texte est soulignée par un système décoratif très caractéristique du Miroir historial ${ }^{5}$. La forte personnalisation de ces manuscrits s'intègre également dans le décor et en respecte le caractère hiérarchique. Outre l'armorial qui occupe la totalité d'une

2. Voir C.Ranvier, L'Iconographie mariale et christique $d u$ Miroir historial $(B n F$ Français 50) réalisé pour Jacques d'Armagnac durant la seconde moitié du XV siècle, mémoire de Master, Université Paris Ouest Nanterre La Défense, Paris, 2010.

3. Voir la généalogie textuelle des différentes versions du Miroir historial dans L. Brun \& M. Cavagna, «Pour une édition du Miroir historial de Jean de Vignay», Romania, 124 (2006), p. 378-428, et C. Ranvier, L'Iconographie mariale et christique..., p. 48.

4. Le texte latin original ainsi que les premières versions de ce texte en français étaient divisés en quatre parties. Malgré l'abandon de cette pratique au cours du xiv ${ }^{\mathrm{e}} \mathrm{s}$. et la liberté divisés en quatre parties. Malgré l'abandon de cette pratique au cours du xive $\mathrm{s}$. et la liberté
alors laissée au commanditaire de choisir le nombre de volumes dédiés à la transcription, alors laissée au commanditaire de choisir le nombre de volumes dédiés à la transcription,
la césure textuelle initiale en deux grandes parties subdivisées en deux autres de moindre mportance a été conservée.

5. C.A.Chavannes-Mazel, «Expanding Rubrics for the Sake of a Layout: Mise-en-page as Evidence for a particular Scribe ?», dans L. L. Brownrigg éd., Medieval Book Production: Assessing the Evidence. Proceedings of the Second Conference of the Seminar in the History of the Book to 1500 (Oxford, July 1988), Los Altos Hills, 1990, p. 117-131. page en frontispice du second volume, le bas de chaque page dotée d'un encadrement de végétaux présente l'armorial du duc de Nemours: l'écu, tenu par deux sirènes, est timbré d'un lambrequin d'azur et de gueules ainsi que d'un casque, lui-même surmonté de la couronne ducale et d'une gerbe de blé, et repose sur un lion couché. Des lambrequins sont déployés audessus de l'ensemble grâce à une corde par deux hommes sauvages munis d'un gourdin qui flanquent les tenants de l'armorial ${ }^{6}$. L'écu est également peint sur les trois tranches de chaque volume. L'anagramme de la devise du duc de Nemours, «fortune d'amis», apparaît sur les phylactères tenus par les êtres hybrides qui ornent ces mêmes encadrements.

Cette fastueuse illustration aux couleurs lumineuses très largement rehaussées d'or bruni fait écho à la reliure exceptionnelle qui, à l'origine, protégeait les trois ouvrages. Six cabochons et quatre cornières de cuivre doré ornaient initialement le velours vermeil qui recouvrait chacun des trois manuscrits dont la fermeture était assurée par deux lanières de cuir gainée d'une étoffe et munies d'une agrafe métallique. Aujourd'hui, seul le dernier volume a conservé sa reliure originale. Son absence sur les deux autres manuscrits et les modifications de certains éléments du décor témoignent des fortunes diverses qu'ont connues ces ouvrages après la mort du duc de Nemours en 1477.

L'arrestation de Jacques d'Armagnac au début de l'année 1476 met fin au siège de sa demeure de Carlat ainsi qu'à près de treize ans de rébellion contre Louis XI. Le crime de lèse-majesté dont le duc de Nemours est accusé dérogeant à la personnalité des peines, ses terres et biens meubles sont confisqués par le roi. Cependant, bien que la Couronne ne prenne officiellement le contrôle des terres du duc qu'en 1489, il semble que la répartition des «dépouilles» ait eu lieu dès son arrestation ${ }^{7}$. Comme il est d'usage à l'époque, ce sont les membres de la commission nommée par le roi pour juger Jacques d'Armagnac qui se sont partagé ses biens ${ }^{8}$, ses terres comme ses livres.

C'est dans ce cadre que les trois volumes du Miroir historial changent de propriétaire, comme en atteste la modification des armoriaux. L'ex-libris autographe, l'écu et la devise de Jacques d'Armagnac des deux premiers manuscrits ont été grattés et remplacés par les armes et la devise de Pierre de Beaujeu, futur duc de Bourbon. Prince fidèle à Louis XI, il fut responsable de la capture du duc de Nemours et présida son procès. Outre une partie de

6. Les armes peintes sur l'écu combinent la charge de la maison d'Armagnac de Castanet et celle de la maison Bourbon-la Marche: au un et quatre, de Bourbon - d'azur à trois fleurs de lis d'or - à la bande chargée de trois lionceaux d'argent, au deux et trois, d'Armagnac - a un et quatre d'argent au lion de gueule, au deux et trois, de gueule au léopard lionné d'or.

7. Le fait est corroboré par l'écu de Tanneguy du Chastel qui apparaît dans le troisième volume du Miroir historial bien qu'il soit mort la même année que Jacques d'Armagnac.

8. A. Sablon du Corail, Louis XI ou le Joueur inquiet, Paris, 2011, p. 343. 
la bibliothèque de Jacques d'Armagnac, il reçut le gouvernement du comté de la Marche et la vicomté de Carlat. En 1527, les deux volumes du Miroir historial qui lui sont échus, tout comme les autres biens des Bourbons revendiqués par Louise de Savoie, intègrent les collections royales mais ne reçoivent une nouvelle reliure que deux siècles plus tard. Ils sont aujourd'hui encore recouverts du maroquin rouge caractéristique des reliures royales du xviiie siècle et sont conservés à la Bibliothèque nationale de France sous la cote fr. 50 et fr. 51 .

Seul l'ex-libris et le premier armorial du troisième volume du Miroir historial du duc de Nemours ont été grattés. En lieu et place apparaissent les armes de Tanneguy du Chastel, vicomte de la Bellière. Selon Émilie Cotterau-Gabillet, bien que ce dernier ait participé à l'arrestation de Jacques d'Armagnac, il semble n'avoir rien reçu lors du partage des biens du duc. Toutefois, Pierre de Beaujeu, qu'il a épaulé durant cette période, lui aurait offert un certain nombre de livres ${ }^{9}$. On peut donc supposer qu'à l'origine les trois manuscrits du Miroir historial ont été donnés à Beaujeu. Il aurait ensuite offert le troisième à du Chastel, qui aurait voulu y apposer sa propre marque en faisant repeindre les écus. La mort de du Chastel en 1477 semble cependant avoir interrompu l'opération et le reste du volume conserve les armes d'Armagnac et du Castanet. La trace du manuscrit fut ensuite perdue jusqu'à son achat en 1855 par le duc d'Aumale, qui le conserve au château de Chantilly où il se trouve encore aujourd'hui, recouvert de sa reliure d'origine, sous la cote 722 .

Les ais recouverts de velours protègent la transcription, en français,

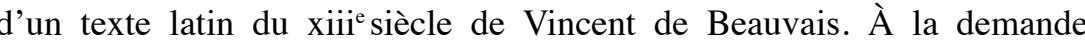
d'Hugues de Saint-Cher, ce lector dominicain entreprit, entre 1230 et 1245, de compiler en un seul ouvrage toutes les choses «dignes de quoy l'on peut avoir regart ou admiracion» afin d'apprendre à «adresser son cuer adieu $»^{10}$. Soumis à l'évolution rapide des savoirs, ce grand miroir du monde - appelé Speculum maius - connut plusieurs réécritures et révisions qui obligèrent Vincent de Beauvais à scinder un texte devenu bien trop long en trois parties. La première, le Speculum naturale, propose une description de la nature suivant l'ordre des six jours de la Création. Les sciences et les arts dont l'homme dispose pour lutter contre les conséquences de sa Chute sont compilés dans la seconde partie, le Speculum doctrinale, tandis que le Speculum historiale retrace le cheminement de l'humanité vers son salut ${ }^{11}$.

9. É.Cottereau-Gabillet, «Procès politique et confiscation: le sort de la bibliothèque de Jacques d'Armagnac» dans F. Foronda, B. Sere et C.Barralis éd., Violences souveraines au Moyen Âge. Travaux d'une école historique, Paris, 2010, p. 242.

10. Extraits des chapitres 3 et 4 du Libellus totius operis apologeticus, prologue du Speculum maius.

11. Voir M.Paulmier-Foucart, M.-C.Duchenne, Vincent de Beauvais et le Grand miroir du monde, Turnhout, 2004.
Vincent de Beauvais a notamment composé la trame historique de ce troisième miroir à partir des Chroniques d'Eusèbe-Jérôme et des citation de Sigebert de Gembloux auxquelles il a ensuite adjoint nombre de textes religieux, tout particulièrement l'Historia scholastica de Pierre Comestor et la Chronique d'Hélinand de Froidmond, ses deux principales sources. Des florilèges d'auteurs païens antiques et des interventions de la main même du lector dominicain complètent ce vaste ensemble. Les grands événements de l'histoire biblique et ecclésiastique y sont rapportés en respectant les jalons chronologiques imposés par l'histoire païenne, dont le récit est complété par de nombreuses digressions littéraires ou hagiographiques. Malgré l'utilisation répétée de textes apocryphes, poétiques ou philosophiques, le Speculum historiale, à l'instar des deux autres parties du Speculum maius, se voulait un texte didactique, un recueil d'exempla à l'attention des futurs prédicateurs de l'ordre dominicain et, de fait, une œuvre strictement religieuse.

La traduction du texte en français par Jean de Vignay, qui intervin autour de 1330, changea radicalement le statut du travail de Vincent de Beauvais. Commandé par la reine Jeanne de Bourgogne, le texte transposé en français quitta la discrétion des réfectoires dominicains où il était lu à haute voix, pour intégrer les collections royales et connaître une diffusion dans un milieu princier et bourgeois auquel il n'était pas destiné. Le phénomène s'explique par ce goût particulier pour l'histoire qui se développa à partir

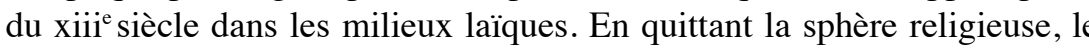
Miroir historial n'était plus considéré comme un livre didactique destiné à la prédication mais comme une vaste fresque historique, un moyen donné à la noblesse de redécouvrir les hauts faits de ses ancêtres. Le contraste saisissant entre les copies du Speculum historiale et celles du Miroir historial illustre parfaitement le changement radical qui s'est opéré lors de cette traduction: à la pauvreté esthétique des transcriptions latines réservées à la formation des frères prêcheurs et à la vie monastique s'oppose la richesse décorative des nombreuses versions françaises destinées à la mise en valeur du mécène. Euvre monumentale abondamment illustrée, la version du Miroir historial commandée par le duc de Nemours le présente à la fois en amateur éclairé, dépositaire d'une culture historique et en homme d'État soucieux d'afficher son pouvoir à la veille de sa disgrâce.

\section{Un manuscrit à l'image de son commanditaire}

Grâce aux recherches effectuées par Chrystèle Blondeau, on connaît le soin apporté par Jacques d'Armagnac à chaque pièce de sa bibliothèque. L'étude des retouches exécutées à sa demande sur le Livre de Lancelot du Lac (Paris, BnF, fr. 117-120) a largement contribué à démontrer sa connaissance 
des grands textes médiévaux et sa volonté de modeler l'illustration de ses manuscrits selon ses exigences de lecteur et d'homme de pouvoir ${ }^{12}$.

De fait, la sélection méticuleuse des parties du Miroir historial destinées à être illustrées parmi 3822 chapitres peut être imputée à Jacques d'Armagnac lui-même. Contrairement à d'autres copies de la traduction de Jean de Vignay dont l'illustration s'avère parfois systématique ${ }^{13}$, la répartition inégale des images dans les trois manuscrits du duc de Nemours indique très clairement un choix volontaire des chapitres destinés à recevoir une miniature. Bien que l'éventualité d'une certaine influence du copiste Gilles Gracien de Poitiers lors de la mise en page du texte soit envisageable, la dominante historique évidente de l'illustration laisse peu de place au doute quant à la participation du commanditaire en personne. L'histoire païenne a en effet été clairement privilégiée lors de l'illustration, à travers la multiplication de scènes de batailles, des représentations de faits de guerre et d'allusions au pouvoir politique, laissant de côté les exégèses et autres discours théologiques. Certains livres se retrouvent littéralement dépourvus d'images, à l'exemple du livre XXIX consacré aux «livres et traicties de saint Bernart abbe de Clairvauz» ou des vingt-trois chapitres qui clôturent le livre VII par des florilèges de poètes païens.

La commande du Miroir historial est-elle donc seulement l'illustration de ce goût pour l'histoire, commun à tous les grands seigneurs $\mathrm{du} \mathrm{xv}^{\mathrm{e}}$ siècle? Bien que l'influence de cette mode historique paraisse indéniable, un examen plus poussé de l'illustration permet de dégager deux grands thèmes, chers à Jacques d'Armagnac, autour desquels s'articule l'iconographie des manuscrits fr. 50, 51 et du manuscrit 722 . Le traitement des scènes à caractère religieux est, à cet égard, tout à fait remarquable. Leur développement au sein d'un récit historique dépourvu de son caractère universel originel - et par conséquent d'une partie de son caractère sacré est en effet particulièrement intéressant. Dans la miniature d'ouverture du Miroir historial, la traditionnelle présentation du livre au commanditaire fait face à une impressionnante allégorie de la construction de l'Église riche de vingt-six personnages. De taille égale et placées côte à côte, les deux scènes sont reliées par un lien visuel évident qui place la réalisation de ce manuscrit au cœur de la foi chrétienne. De la même manière, dans la miniature précédant le livre XXVII (dont la taille exceptionnelle est identique à l'image d'ouverture), la série de rois ainsi que le baptême de Clovis sont littéralement écrasés par une représentation de la mort de Julien l'apostat. Cette scène de bataille apparaît alors comme un prétexte à la représentation

12. C. Blondeau, «Du plaisir des sens à la passion du sens: l'illustration d'un cycle du Lancelot Graal et ses remaniements », dans F. Joubert éd., L'Artiste et le commanditaire aux derniers siècles du Moyen Âge: XIII'-XVI siècles, Paris, 2001, p. 99-114.

13. Notamment les deux premières versions françaises de la fin tragique de cet empereur romain, ainsi nommé après sa tentative de restauration du paganisme. L'écu et l'armorial complet du duc de Nemours, postérieurement remplacés par ceux de Pierre de Beaujeu, sont visibles sur le fronton de l'église qui surplombe cette imposante scène de bataille et créent un lien direct entre le commanditaire et cet événement fondamental de l'histoire religieuse. Enfin, l'Adoration des rois mages (fig. 1) constitue sans doute le meilleur exemple de la foi très forte du duc de Nemours, puisque, à l'instar d'autres grands commanditaires de son époque, il apparaît sous les traits de Gaspard, le plus jeune des mages. L'allusion est claire: des trois rois, il est le seul vêtu à la mode du $\mathrm{Xv}^{\mathrm{e}}$ siècle - chausses, pourpoint à manches pertuisées et poulaines aux pieds -, sans compter sa coiffure, identique à celle qu'il arbore dans la miniature de présentation. Représenté de dos, avançant en direction de la Vierge et de l'Enfant auxquels il apporte un reliquaire doré, il se présente en prince pieux.

Cette piété, particulièrement forte, s'inscrit dans un contexte familial tourné vers le franciscanisme. Dès leur installation à Rodez en 1232, les franciscains ont en effet bénéficié de l'appui des comtes locaux dont ils ont été les confesseurs, parfois les conseillers ${ }^{14}$. Bonne de Berry ou encore Béatrix de Clermont - respectivement grand-mère et trisaïeule de Jacques d'Armagnac - ont marqué l'histoire religieuse locale et très largement participé au rayonnement spirituel du couvent des Cordeliers de Rodez ${ }^{15}$.

Malgré sa vie tumultueuse et bien souvent pécheresse, le duc de Nemours, lui-même membre du Tiers-Ordre à l'instar de ses parents, a toujours entretenu des rapports très étroits avec les franciscains. Il aurait ainsi reçu la tonsure en 1458 et projetait un pèlerinage en Terre Sainte en $1466^{16}$. Fondateur de plusieurs messes, il avait émis le souhait, peu avan son exécution, de voir ses enfants, Jean et Charlotte, ainsi que ses filles bâtardes, confiés à une institution religieuse.

Cet attachement pour l'ordre franciscain est sensible dans l'iconographie du Miroir historial. Outre les thèmes religieux développés dans toutes les miniatures de grande taille, l'influence franciscaine est parfois palpable dans des images particulières, comme le Portement de croix (fig. 4). Placé au premier plan d'une miniature du manuscrit fr.50, ce thème sur lequel saint François a fondé son ordre - «prends ta croix et suismoi », disait-il - relègue la crucifixion dans un arrière-plan peu visible. Cet

14. Guy Brianson, confesseur et astrologue de Jacques d'Armagnac, était lui-même cordelier: cf. J.Blanchard, Procès de Jacques d'Armagnac : édition critique du ms. 2000 de la bibliothèque Sainte-Geneviève, Genève, 2012, p. LXVI.

15. V. Bouat, Les Ordres mendiants et les pouvoirs à Rodez (XIV'-XVI' siècles), thèse de doctorat, École nationale des Chartes, Paris, 2006.

16. Projet annulé suite aux prédictions de Brianson qui le voyait s'y noyer mais égalemen devant la menace d'être assassiné en chemin par les hommes du roi : $c f$. J.Blanchard, Procès de Jacques d'Armagnac..., p. 477 et 484. 
aménagement n'est ni le fait des miniaturistes, dont les modèles d'atelier indiquent que la crucifixion était habituellement peinte seule ou au premier plan, ni issue d'une tradition iconographique liée à l'ouvrage lui-même. Elle émane donc d'une demande spécifique du commanditaire qui, en tant que franciscain, portait très probablement à cette image une attention toute particulière.

Aussi pieux fût-il dans la sphère privée, Jacques d'Armagnac se montrait davantage encore avide de reconnaissance et de pouvoir, un thème perceptible en filigrane à travers toute l'iconographie du Miroir historial, y compris dans des miniatures à dominante religieuse. Même le cycle marial et christique est l'occasion d'évoquer le pouvoir civil. La comparution du Christ devant Hérode Antipas et Ponce Pilate remplace par exemple l'introduction de Jésus devant les représentants du pouvoir religieux, Anne et Caïphe, épisode pourtant développé dans le texte de Vincent de Beauvais. De même, l'arrivée de Marie et Joseph à Bethléem est cantonnée dans une partie exiguë d'une autre image, la représentation de la mise en scène du pouvoir sous les traits d'Hérode ordonnant le cens devenant alors le sujet central de la miniature.

L'omniprésence de la thématique du pouvoir n'est pas sans rappeler la position politique du duc de Nemours lui-même. La date de la commande du Miroir historial - 1459 - correspond à l'apogée de la carrière politique de Jacques d'Armagnac. Comte de la Marche, de Castres et de Pardiac, vicomte de Carlat et de Murat dont il hérite, il obtient en 1461 le duché de Nemours lorsque Charles VII, peu avant sa mort, renonce à ses droits sur ces terres. Devenu pair de France à double titre, il épouse en 1462 Louise d'Anjou, cousine et filleule de Louis XI, ce qui fait de lui rien de moins que le cousin du roi. La même année, ce dernier tranche en faveur de Jacques d'Armagnac un conflit entre les maisons de Bourbon et de Navarre concernant le duché-pairie de Nemours puis le nomme lieutenant en Roussillon en $1463^{17}$.

Le «mignon» du roi, tel que l'ont surnommé les chroniqueurs de l'époque, est devenu, à l'aube de cette année-charnière, l'un des seigneurs les plus puissants de son temps grâce aux faveurs accordées par les souverains, Charles VII puis Louis XI. Il n'est donc pas surprenant que sa première commande bibliophilique soit jalonnée de références au pouvoir puisqu'elle en est l'un des symboles. La multiplication des marques de possession dans le Miroir historial contribue à l'affirmation de cette nouvelle autorité. On ne compte ainsi pas moins de trente-six armoriaux, dont le plus grand - en frontispice du second volume - occupe un feuillet entier, complétés par les écus disséminés çà et là dans différentes miniatures, les ex-libris autographes, aujourd'hui disparus et les anagrammes de la devise de Nemours. Même

17. Cf. J.Blanchard, Procès de Jacques d'Armagnac..., p. X-XI les éléments constitutifs des trois manuscrits mettent en scène la puissance de Jacques d'Armagnac. Les plus beaux matériaux ont été employés pour honorer cette formidable commande. Outre le cuivre doré, utilisé pour les cornières, fermoirs et autres cabochons, on remarque l'usage du velours - l'étoffe la plus prestigieuse - coloré en vermeil, la teinture la plus chère de l'époque. De même, indépendamment de l'or utilisé en grande quantité pour le travail d'enluminure, l'éclat actuel des couleurs utilisées pour la décoration témoigne de la qualité des pigments employés alors. Et que dire des 1350 feuillets de parchemin qui ont nécessité l'abattage de près de 675 veaux!

Les moyens financiers mis en œuvre pour la réalisation de ce triple manuscrit et la participation du duc de Nemours à l'élaboration des thématiques, voire à l'iconographie de certaines scènes, témoignent de l'importance qu'il revêtait aux yeux du commanditaire. De fait, choisir le texte de Vincent de Beauvais pour ce premier acte de mécénat ne pouvait être anodin. Il n'est pas impossible que ce choix ait été dicté par la présence d'un autre manuscrit dans la bibliothèque de Jacques d'Armagnac: le premier des quatre volumes d'un autre Miroir historial. Actuellement conservé à la Bibliothèque nationale de France sous la cote fr.316, ce volume a reçu le qualificatif de «J1» de la part de Claudine A. Chavannes-Mazel dans son étude sur le Miroir historial de Jean le Bon (dans laquelle les différentes versions françaises de ce texte ont fait l'objet d'une codification $)^{18}$. Jacques d'Armagnac a vraisemblablement hérité de $\mathrm{J} 1$ autour de 1463, à la mort de sa mère Éléonore de Bourbon, soit près de quatre ans après la commande de son propre Miroir historial. On sait toutefois qu'Éléonore de Bourbon a passé les dernières années de sa vie dans la demeure de son fils. Sa bibliothèque l'ayant sans doute accompagnée, on peut raisonnablement penser que Jacques d'Armagnac a été mis en contact avec J1 avant 1459 et que ce manuscrit a joué un rôle décisif dans la définition de son premier projet bibliophilique. Mais comment un manuscrit qui ne contenait qu'un quart du texte aurait-il pu influencer Jacques d'Armagnac ${ }^{19}$ ?

La série $\mathrm{J}$ est exceptionnelle car elle constitue, avec la série jumelle «A», la première transcription française connue du texte de Vincent de Beauvais. Il s'agit de la commande de Jeanne de Bourgogne faite autour de 1330 à l'occasion de deux événements concomitants: le mariage de sa fille et l'adoubement de son fils ${ }^{20}$. Jacques d'Armagnac a pu hériter de J1

18. C. A.Chavannes-Mazel, The Miroir historial of Jean le Bon. The Leiden Manuscript and his related Copies, Cambridge, $2^{\text {de }}$ édition, 1991

19. On sait notamment que $\mathrm{J} 2$, le second volume de cette série, a disparu au début du $\mathrm{xv}^{\mathrm{e}}$ siècle. J3 et J4 étaient alors également perdus. Des quatre volumes, seuls J1 et J3 (réapparu à Baltimore) ont été retrouvés.

20. Le texte de la série A a été révisé, contrairement à celui de la série J, et l'enluminure a été confiée à deux historieurs différents. Le seul point commun de ces deux versions du Miroir 
grâce à sa filiation avec Jean de Berry, son bisaïeul, lui-même petit-fils de Jeanne de Bourgogne. Or, on sait avec quelle admiration le duc de Nemours considérait son illustre arrière-grand-père, et avec quelle attention il a tenté de se placer dans la droite ligne de celui que l'on considérait déjà à l'époque comme l'un des plus grands bibliophiles de son temps ${ }^{21}$. La commande du Miroir historial constitue très probablement la première tentative du duc de Nemours pour se rapprocher de son modèle.

La confrontation entre $\mathrm{J} 1$ et la première commande de Jacques d'Armagnac est autrement plus intéressante lorsque l'on considère l'image qui ouvre J1. La miniature de présentation montre une double scène où, d'une part, Louis IX commande le Speculum historiale à Vincent de Beauvais, alors que d'autre part, Jeanne de Bourgogne en commande la traduction en français à Jean de Vignay ${ }^{22}$. Cette image est cependant un contresens historique puisque l'ordre dominicain est le véritable commanditaire de la version latine. Toutefois, Louis IX a réellement joué un rôle fondamental dans la naissance du texte. Véritable soutien moral et financier, le saint roi a favorisé l'accès de Vincent de Beauvais aux plus grandes bibliothèques du royaume tout en lui fournissant l'argent nécessaire à la mise au propre de son travail ${ }^{23}$.

Le Speculum historiale n'est toutefois pas exempt de toute référence politique puisque le chapitre 126 du livre XXXI - Reditus ad stirpem Caroli-, probablement ajouté à la demande de Louis IX en 1244, souligne la continuité entre les Carolingiens et les descendants d'Hugues Capet ${ }^{24}$. L'erreur historique développée dans la miniature de J1 est tout à fait volontaire et témoigne de la période d'instabilité politique durant laquelle le texte a été traduit, tandis qu'Édouard III d'Angleterre, neveu des trois précédents rois de France, revendique le trône de Philippe VI. Édouard III, petit-fils de Philippe le Bel, se disait alors plus proche des Capétiens que Philippe VI, cousin du dernier Capétien direct ${ }^{25}$. L'image qui ouvre J1, hautement symbolique, doit se comprendre comme un jeu de miroir destiné à placer Jeanne de Bourgogne, petite-fille de Louis IX et épouse de l'arrière-

historial ne concerne donc que leur commande simultanée par un même commanditaire. Voir C. A. Chavannes-Mazel, «Problems in Translation, Transcription and Iconography: the Miroir historial, Books 1-8», dans M.Paulmier-Foucart, S. Lusignan, A. Nadeau éd., Vincent de Beauvais, intentions et réceptions d'une ceuvre encyclopédique au Moyen Âge, Actes du XIV colloque d'études médiévales, Montréal, 27-30 avril 1988, Montréal, 1990 , p. 345-374

1. Voir C.Blondeau, «Du plaisir des sens...», p. 101.

22. Une symétrie visuelle souligne le lien entre les deux souverains par l'emploi probable d'un même modèle.

23. S. Lusignan, Préface au Speculum Maius de Vincent de Beauvais, réfraction et diffraction, Montréal-Paris, 1979, p. 57-58.

24. M. Paulmier-Foucart \& M.-C.Duchenne, Vincent de Beauvais..., p. 82-83.

25. C. Beaune, Naissance de la nation France, Paris, 1993, p. 267. petit-fils du saint roi, dans la droite ligne de son aïeul, légitimant ainsi le couple royal en se référant au plus illustre des Capétiens.

Jacques d'Armagnac ne pouvait ignorer la portée politique et l'idée de continuité monarchique véhiculée par cette image, d'autant plus qu'i était lui-même un descendant de SaintLouis de par son lien de parenté avec Jean de Berry, Philippe VI de Valois et Jeanne de Bourgogne. Le fait est d'autant plus clair que la miniature d'ouverture du manuscrit fr. 50 présente également deux scènes mises en regard. La version du Miroir historial commandée par le duc de Nemours, dont le texte a acquis une dimension royale, peut donc être considérée comme une démonstration de sa puissance grâce à l'affirmation de son illustre ascendance, qui le relie à la fois au plus respecté des souverains et au plus grand bibliophile de son temps.

On peut néanmoins raisonnablement se demander quelle fut la portée de ce projet bibliophilique à l'aube de l'année 1463, lorsque la vie politique de Jacques d'Armagnac fut brutalement bouleversée. Seigneur fidèle et ami de Louis XI, à qui il devait une grande partie de sa fortune, le duc de Nemours changea radicalement de position vis-à-vis de son souverain au retour de l'expédition de Roussillon qu'il mena victorieusement pour le roi en 1462. À défaut de récompenser le duc pour sa réussite, Louis XI, connu pour son excessive piété, choisit de faire un don de cent écus à la châsse de Saint-Martin de Tours, sans rembourser Jacques d'Armagnac des frais qu'il avait engagés. Ce malheureux incident marqua le point de départ de la trahison de ce dernier, qui entra en pourparlers avec Antoine de Chabannes dès 1464, avant de rejoindre avec lui la Ligue du Bien Public pour lutter contre le roi. Malgré le pardon de ce dernier, le duc de Nemours ne cessera de se laisser entraîner dans une série de complots et de trahisons - notamment en faveur de Charles de France, frère cadet du roi - en dépit de son serment d'allégeance au souverain, renouvelé par trois fois mais toujours bafoué. Louis XI, excédé, le fit arrêter en 1476. Accusé, entre autres, d'avoir participé à un projet de capture puis d'empoisonnement du roi, d'avoir empêché les agents royaux de faire leur travail sur ses terres ou encore «d'anglicherie», à cette époque où les Anglais venaient de débarquer à Calais (1475), le «mignon» du roi - devenu «le petit Judas» - fut finalement condamné pour crime de lèse-majesté et décapité le 4 août 1477 sur la place des Halles à Paris ${ }^{26}$.

L'année 1463 correspond à la fois à la prise de position radicale du duc de Nemours contre le roi et à la fin de la transcription du Miroir historial et, de fait, au début de sa mise en image.

On sait, grâce au travail de Chrystèle Blondeau, que Jacques d'Armagnac n'a pas hésité à critiquer le roi à travers ses commandes

26. J. Blanchard, Procès de Jacques d'Armagnac... 
$\operatorname{artistiques}^{27}$. À cet égard, si sa première commande n'apparaît être que la démonstration de puissance d'un seigneur en pleine gloire, elle peut être considérée après 1463 comme une bravade envers le souverain. L'utilisation de l'image de Louis IX n'est en effet pas anodine à la fin du Moyen Âge. Saint modèle jusqu'à l'avènement de Charles VII, puis de Louis XI - qui lui préfèreront la symbolique religieuse et guerrière de saint Michel - Saint Louis bénéficie auprès des princes $\mathrm{du} \mathrm{xv}^{\mathrm{e}}$ siècle de l'image, vivace bien qu'erronée, du protecteur des nobles contre le développement de l'autorité royale ${ }^{28}$. Transposée dans le cadre de la Ligue du Bien Public qui luttait contre l'emprise de la souveraineté royale que Louis XI tentait d'imposer alors, la référence à saint Louis devient politiquement lourde de sens.

Certaines images du Miroir historial peuvent également poser question. La représentation du duc de Nemours en roi mage n'est-elle révélatrice que d'une profonde piété ? Aucun des peintres travaillant dans l'atelier chargé de la mise en image des trois manuscrits n'avait jusqu'à cette date utilisé un modèle identique à celui du Miroir historial (fig. 1). Il leur a fallu combiner deux modèles, l'un pour la figure de Gaspard - notamment réutilisé par Maître François dans un manuscrit de la Cité de Dieu de saint Augustin $^{29}$ - et un second pour le reste de la scène, laissant pressentir une possible demande spécifique du commanditaire. Ce roi religieux, coiffé comme le duc de Nemours dans la miniature d'ouverture, portant habits de l'époque et si curieusement représenté de dos qu'on ne peut voir les traits de son visage, ne pourrait-il être lu comme un pied de nez au roi terrestre ${ }^{30}$ ?

Enfin, d'aucuns considèrent que la représentation de Tibère III donnant sa fille Constance en mariage à Maurice - peinte dans le troisième volume de cet ensemble - fait allusion au propre mariage de Jacques d'Armagnac et Louise d'Anjou ${ }^{31}$, théorie d'autant plus plausible que les personnages sont tous vêtus à la mode du $\mathrm{Xv}^{\mathrm{e}}$ siècle. Sachant que, par ce geste, Tibère III désigne Maurice comme son successeur, cette miniature peut à son tour revêtir une signification politique très forte.

Placée dans ce contexte particulier, la reliure, seul élément dont on sait avec certitude qu'il fut réalisé bien après l'année-charnière 1463, peut également être considérée comme un outil de propagande. S'il est vrai que le velours vermeil pouvait recouvrir les manuscrits les plus précieux d'une riche collection, de telles couvertures étaient l'usage de la maison royale de France. Si la commande d'un livre royal permettait à Jacques d'Armagnac de s'imposer en seigneur puissant, il est fort probable que ce dernier ait

27. Voir C. Blondeau, «Du plaisir des sens...», p. 112-114.

28. C. Beaune, Naissance de la nation France..., p. 140-147.

29. Paris, Sainte Geneviève, $246, f^{\circ} 232 v^{\circ}$

30. C. Ranvier, L'Iconographie mariale et christique..., 2010, p. 149-153.

31. I. Delaunay, J. B.Lebigue, S. Lefèvre et al., L'Enluminure en France au temps de Jean Fouquet, Paris, 2003, p. 66 cherché, au fil du temps, à égaler son souverain en utilisant un vocabulaire symbolique couramment utilisé par la royauté ${ }^{32}$. Lorsque l'on sait qu'en 1465, en cas de victoire de la ligue du Bien Public, le roi devait être mis sous tutelle de Jacques d'Armagnac, ces références au pouvoir royal paraissent chargées d'un poids politique certain.

\section{Le travail de l'atelier parisien}

Pour exécuter les quelques cinq cents miniatures prévues dans ces trois manuscrits monumentaux, le duc de Nemours a fait appel à un atelier parisien ${ }^{33}$. La capitale sinistrée, libérée du joug anglais depuis 1436 , se remet alors lentement de cent ans de guerre, de crises économiques, d'épidémies et de luttes intestines entre Bourguignons et Armagnacs. Après le règne de Charles VII, qui a délaissé Paris au profit d'autres villes telles que Bourges, divers foyers artistiques émergent, privant la capitale du statut central qu'elle occupait auparavant malgré des mesures visant à attirer les $\operatorname{artistes}^{34}$. La société tout entière est en pleine mutation: la commande artistique n'est plus l'apanage des princes de sang et autres membres de la cour. Les clients issus de la haute bourgeoisie se multiplient: officiers, chefs militaires, prélats, noblesse de robe, etc.

C'est dans une capitale en pleine reconstruction que commence la décoration du Miroir historial de Jacques d'Armagnac, dans l'un des ateliers les plus productifs de l'époque. On connaît trois historieurs ayant travaillé dans cette officine au cours du xv ${ }^{\mathrm{e}}$ siècle: le Maître de Rolin ${ }^{35}$ - qui tire son nom de plusieurs travaux exécutés pour le cardinal Jean II Rolin -, Maître François - connu pour la mise en image de plusieurs manuscrits de la Cité de Dieu de saint Augustin - et le Maître de Jacques de Besançon.

Nos premières conclusions ont montré que la participation du duc à l'élaboration technique et picturale de son Miroir historial, bien qu'avérée,

32. En cela Jacques d'Armagnac n'est pas différent des autres commanditaires de la fin du Moyen Âge. Des princes aux bourgeois, tous sont préoccupés par leurs propres représentations et ont besoin de référents. Les œuvres qu'ils commandent constituent, pour la plupart, une «littérature de propagande soumise à [leurs] intérêts »: $c f$. B. Guenée, Histoire et culture historique dans l'Occident médiéval, Paris, 1980, p. 64; F. Joubert dir., L'Artiste et le commanditaire aux derniers siècles du Moyen Âs, 1980, p. 6

33. Il ambitionnait le gouvernement de Paris et de l'Île-de-France, qu'il n'obtiendra finalement pas (J.Blanchard, Procès de Jacques d'Armagnac..., p. XIII). On notera au passage le choix d'un atelier de la ville royale pour la décoration de ce livre politiquement tendancieux

34. En 1443, une exemption d'impôt est proposée à tout artiste venant s'installer à Paris. Voir F.Avril \& N. Reynaud, Les Manuscrits à peintures en France (1440-1520), Paris, 1993.

35. Le Maître de Rolin était initialement connu sous le nom du Maître de l'horloge de Sapience. Voir P. R. Monks, The Brussels Horloge de Sapience, Leiden, 1990. 
reste ponctuelle. De fait, on peut considérer que les peintres ont bénéficié d'une certaine liberté dans l'exécution des miniatures. La comparaison du cycle marial et christique du fr.50 avec la production très large de ces historieurs dans cette même thématique - particulièrement populaire à la fin du Moyen Âge - permet d'apprécier l'usage, relativement réduit, que les peintres ont fait de cette liberté offerte par le commanditaire.

Les miniatures ne présentent en effet que peu d'innovations iconographiques. Les architectures et les paysages urbains sont standardisés, les personnages négatifs tels que le peuple juif dans le cycle de la vie du Christ apparaissent comme il est alors d'usage, dépréciés par leurs costumes, qu'ils soient jaunes, bariolés, incomplets ou analogues à celui des musulmans. Les peintres ont toutefois assimilé certaines innovations iconographiques de leur époque, notamment la multiplication des personnages ou la représentation des nouveaux thèmes caractéristiques de la sensibilité de la fin du Moyen Âge. Par exemple, le thème, récemment apparu, du Procès de Paradis met l'accent sur l'inadéquation partielle entre les peintures et le texte qu'elles sont censées illustrer, puisqu'il s'agit d'une image issue du théâtre liturgique de l'époque, donc absente de l'imagerie du xiiie siècle. Les miniaturistes n'ont donc pas pris connaissance du texte qu'ils devaient historier. Cette hypothèse se précise lorsque, à l'inverse, une miniature correspond au texte qu'elle illustre, car il s'agit alors presque systématiquement d'un passage dans lequel Vincent de Beauvais a repris les textes apocryphes - ces mêmes textes qui ont servi au développement d'une nouvelle iconographie du $\mathrm{xv}^{\mathrm{e}}$ siècle. En outre, certains passages textuels pouvaient être connus des enlumineurs grâce à la diffusion de la Légende dorée de Jacques de Voragine ${ }^{36}$

Les historieurs ont vraisemblablement fondé leur travail sur la simple lecture des rubriques bien plus que sur celle du texte afin de placer correctement et rapidement des images génériques, réutilisées dans le cadre de livres d'heures et d'autres ouvrages religieux. Cette possibilité est d'autant plus envisageable que la récurrence de nombreux motifs semble indiquer l'usage de modèles d'atelier.

Ces modèles comprennent à la fois des motifs réutilisables par les historieurs dans différentes peintures et des guides génériques qui proposent des exemples de scènes entières. On retrouve toutes ces occurrences dans le cycle marial et christique du Miroir historial de Jacques d'Armagnac. Les motifs d'anges en forme de virgule, placés à l'arrière-plan, se multiplient à travers le cycle en présentant souvent exactement la même position (fig. 2).

36. On ne peut, en effet, imaginer que les enlumineurs aient eu connaissance, par exemple, de l'anecdote de la guérison du roi Abgar par saint Jude grâce au premier texte qui en fait mention, l'Historia scolastica de Petrus Comestor. L'utilisation d'une littérature de substitution plus populaire, à l'instar de la Légende dorée de Jacques de Voragine, parait plus probable.
Ces guides étaient partagés par tous les historieurs de l'atelier qui les modifiaient parfois selon leur goût (fig. 2 et 3 ). Les scènes de Pentecôte du Maître de Rolin, par exemple, sont caractérisées par une composition centrée autour de la Vierge tandis que Maître François adoptait toujours un schéma symétrique en opposant les apôtres à la figure mariale ${ }^{37}$. Les différences entre les scènes d'Annonciation sont plus ténues: Maître François préférait placer la Vierge devant un lutrin plat ou d'un seul tenant, tandis que le Maître de Rolin choisissait un lutrin en deux parties avec un pupitre oblique ${ }^{38}$. En revanche, plusieurs modèles des deux maîtres étaient rigoureusement identiques, notamment la scène de l'Arrestation du Christ (fig. 2 et 3). La combinaison de carnets de motifs et de guides génériques était également chose courante. Outre l'exemple de l'Adoration des Mages (fig. 1) que nous avons déjà évoqué, la scène présentant Auguste ordonnan le cens combine à la fois un personnage placé sous un dais - caractéristique de la représentation commune de l'autorité chez les trois historieurs de l'atelier -, l'ange en virgule et le groupe de Marie et Joseph habituellement utilisé pour les scènes de la fuite en Égypte.

Par ailleurs, l'archaïsme iconographique de certaines scènes, comme la Flagellation du Christ ou le Couronnement d'épines (fr. 50 , fo 230 ), induit l'utilisation de modèles plus anciens. Paul Durrieu, à la fin du xix ${ }^{\mathrm{e}}$ siècle, puis Eleanore Spencer, dans les années 1930, ont relié la production du Maître de Rolin, de Maître François et du Maître de Jacques de Besançon à celle, plus ancienne, du Maître de Bedford ${ }^{39}$. Précédent responsable de cet atelier, le Maître de Bedford a probablement légué ses carnets de motifs à ses successeurs, qui les ont largement repris en les remettant parfois à la mode iconographique de leur époque. C'est la raison pour laquelle on retrouve une scène de Portement de croix dans le Codex 1840 de la Österreichische Nationalbibliothek de Vienne illustré par le Maître de Bedford (fig. 5) similaire à celle du Miroir historial (fig. 4). Pour l'exécution de l'image du manuscrit du duc de Nemours, il a suffi au peintre de multiplier les personnages en ajoutant les saintes femmes et les deux larrons et de basculer la figure du Christ portant la croix. Cette dernière modification démontre, à elle seule, l'assimilation de l'évolution de la piété de la fin du Moyen Âge par la production d'un art douloureux qui dépeint, parfois avec force détails, les tourments du Christ avant sa crucifixion. La figure

37. Voir par exemple le ms. Add. 25695 de la British Library, fo 184 , pour le Maître de Rolin, et le ms. 5154 de la BM de Lyon, $\mathrm{f}^{\circ} 148$, pour Maître François.

38. Le Maître de Jacques de Besançon, très productif mais peu inventif, reprenait d'une manière générale tous les modèles utilisés par Maître François, dont il était très probablement l'élève.

39. P. Durrieu, Un grand enlumineur parisien au XVesiècle: Jacques de Besançon et son ceuvre, Paris, 1892; E. A.Spencer, Maître François and his atelier, Ph. D., Harvard University, 1931. 
maladroitement basculée, supposée matérialiser la chute du Christ sur le chemin de croix, n'a cependant subi aucune autre modification, que ce soit au niveau des mouvements du personnage ou des plis de sa robe, ce qui lui donne un aspect pour le moins hiératique. Quant au sonneur de busine, que le Maître de Bedford a représenté à cheval, il est en partie caché par la figure de Simon de Cyrène dans le Miroir historial, évitant ainsi au peintre le fastidieux dessin du cheval.

De fait, l'organisation de cet atelier parisien donne aux artistes qui y officient les moyens de travailler relativement vite: ils savent quelle scène placer dans les espaces réservés par le copiste grâce aux rubriques du texte et s'appuient sur un vocabulaire iconographique générique composé à l'aide de modèles d'ateliers parfois anciens qu'ils adaptent au cadre, à la demande ou selon leur goût. Toutefois, si la structure de l'atelier permet une production soutenue, elle laisse peu de place à l'originalité et à la personnalité des artistes.

De fait, l'historiographie démontre que l'utilisation systématique de modèles iconographiques par les différents historieurs de l'atelier rend l'attribution de leur travail malaisée. Car si la littérature est relativement abondante sur le sujet, celui-ci fait débat depuis plus d'un siècle.

L'histoire de la découverte de cet atelier d'enluminure parisien débute en 1892, lorsque le comte Paul Durrieu rassemble un corpus de manuscrits, auquel appartient le Miroir historial du duc de Nemours ${ }^{40}$. P. Durrieu attribue la peinture de la totalité des ouvrages à Jacques de Besançon, dont le nom apparaît dans le colophon de l'un des volumes qu'il a rassemblés. Six ans plus tard, Louis Thuasne découvre une lettre de Robert Gaguin dans laquelle est mentionné un manuscrit de la Cité de Dieu enluminé par un certain «egregius pictor franciscus». Cet ouvrage de saint Augustin appartenant à son corpus de manuscrits, P. Durrieu se voit obligé de réviser son travail et attribue finalement, entre 1910 et 1915, le groupe complet à ce «pictor franciscus », francisé en Maître François, sans plus tenir compte du colophon sur lequel il avait précédemment fondé son travail. Il faut attendre les années 1930 et la thèse d'Eléanore P.Spencer pour que ce corpus bénéficie enfin d'une étude systématique qui permet de distinguer trois enlumineurs dont le style, extrêmement proche, justifie leur appartenance au même atelier, dans des tranches chronologiques toutefois différentes ${ }^{41}$. Maître François, un temps considéré comme le fils de Jean Fouquet avant d'être assimilé au peintre berruyer François Colombe, fut

40. P. Durrieu, Paris, 1892

41. Maître François aurait ainsi succédé au Maître de Rolin - le plus âgé - et formé le Maître de Jacques de Besançon, le plus jeune. enfin confondu avec un artiste attaché au comte du Maine. Sa production exclusivement parisienne, a finalement invalidé toutes ces théories ${ }^{42}$.

Bien que particulièrement prolifique, Maître François ne serait pas, selon Eleanore Spencer, l'historieur du Miroir historial de Jacque d'Armagnac. Des comparaisons stylistiques et iconographiques ont rapidement amené la chercheuse américaine à attribuer ces images au «groupe d'Autun» qui sera par la suite réduit à la figure du Maître de Rolin ${ }^{43}$. Le travail d'Eleanore Spencer, fondamental bien qu'ancien, semble avoir été méconnu ou ignoré par les chercheurs suivants qui ont continué d'attribuer l'illustration des manuscrits fr.50, fr.51 et du manuscrit 722 à Maître François ${ }^{44}$.

Par ailleurs, on peut s'étonner de voir des spécialistes attribuer un travail aussi titanesque à un seul peintre. En 1993, Susan A.Blackman a suggéré que pas moins de trois mains pouvaient être différenciées, chaque artiste ayant puisé dans les mêmes carnets de modèles ${ }^{45}$. Elle n'a toutefois pas proposé d'attribution. On peut très logiquement penser que les trois maîtres de l'atelier correspondent à ces trois mains mais aucun travail n'avait jusqu'à présent permis de valider cette hypothèse. Les variations dans l'iconographie et l'utilisation des modèles d'atelier par chaque artiste ayant travaillé sur le Miroir historial permettent pourtant de leur en attribuer les images, parfois même très précisément. À cet égard, l'étude du cycle marial et christique donne à nouveau de nombreux indices.

La composition centrée de la Pentecôte dans le Miroir historial et le lutrin en deux parties visible dans l'Annonciation sont deux élément caractéristiques de l'iconographie du Maître de Rolin. On sait également que ce dernier laissait souvent les éléments architecturaux dépasser du cadre qui circonscrivait l'image ${ }^{46}$, ce qui lui confère d'emblée la responsabilité de plusieursillustrations, notamment Auguste ordonnant le cens. Une conclusion similaire peut être tirée de l'observation de l'Adoration des mages. Dans les premières images du cycle marial, Joseph est un vieillard barbu dont le crâne chauve est coiffé d'un toupet. Il s'agit du type iconographique utilisé pour représenter Melchior dans l'Adoration des Mages alors que Joseph y apparaît beaucoup plus jeune (fig. 1). Maître François n'hésitait pas à attribuer le même visage aux deux personnages dans cette scène, tandis que le Maître de Rolin évitait ce problème en cachant le visage de Joseph derrière un élément architectural ou supprimait simplement le personnage

42. F. Avril, N. Reynaud, Les Manuscrits à peinture..., p. 45

43. La participation du maître de Jacques de Besançon a été d'office écartée car sa période d'activité, qui s'étend sur toute la fin du $\mathrm{xv}^{\mathrm{e}}$ siècle, semblait trop tardive.

44. F. Avril, N. Reynaud, Les Manuscrits à peinture...

45. S. A.Blackman, The Manuscripts and Patronage of Jacques d'Armagnac..., p. 152.

46. P. R.Monks, The Brussels Horloge de Sapience..., p. 20. 
entier de la scène ${ }^{47}$. Si Maître François avait peint l'Adoration des Mages du manuscrit fr. 50, Melchior et Joseph seraient semblables. On peut donc raisonnablement penser que cette image est née sous le pinceau du Maître de Rolin.

S'il semble que ce dernier ait très largement travaillé sur ce cycle, doit-on pour autant écarter la participation de Maître François? Probablement pas. On retrouve en effet plusieurs caractéristiques structurelles et iconographiques qui lui sont propres. Il est, par exemple, le seul des trois historieurs de l'atelier à placer différentes scènes d'une même image dans des registres strictement séparés et superposés ${ }^{48}$. La miniature du Miroir historial présentant conjointement la Cène, le Lavement de pieds et l'Institution de l'Eucharistie, selon cette hiérarchie, indique que l' " egregius pictor franciscus» a effectivement contribué à l'illustration du Miroir historial du duc de Nemours. Un élément iconographique est cependant plus révélateur encore de sa participation: les nimbes. Le Maître de Rolin nimbait ses figures saintes d'un cercle d'or plein rehaussé d'une coquille à l'encre noire, dont le motif différait pour la figure du Christ (fig. 4 et premier plan de la fig. 2). Maître François, quant à lui, superposait à l'or bruni une fine croix rouge sur le nimbe du Christ et laissait les autres vierges de tous motifs (arrière-plan de la fig. 2 et fig. 3).Cette caractéristique iconographique systématique est déterminante et démontre qu'il a entièrement peint trois miniatures complètes du cycle marial et christique du fr. 50 mais qu'il a également exécuté des scènes secondaires.

Il est néanmoins possible d'affirmer qu'il a participé à l'exécution des autres miniatures. Les anges en virgule, les mandorles à fond rouge et les architectures aux tours crénelées sont des détails iconographiques propres à Maître François qu'il a utilisés en abondance dans les manuscrits de la Cité de Dieu et que l'on retrouve dans les arrière-plans du cycle marial et christique du fr. 50 .

Cette particularité permet de dégager quelques principes relatifs aux méthodes de travail de l'atelier. Maître François était manifestement responsable de la réalisation des arrière-plans - paysages, ciels, villes mais aussi scènes secondaires - tandis que le Maître de Rolin travaillait exclusivement sur les scènes principales. Il apparaît alors qu'au début de la mise en image du Miroir historial de Jacques d'Armagnac, Maître François n'était pas encore ce chef d'atelier décrit par Nicole Reynaud. Cette responsabilité était logiquement dévolue au Maître de Rolin, plus âgé.

47. Comparer par exemple le fo 95 du ms. Egerton 2045 de la British Library au fo $27 \mathrm{v}^{\circ}$ du ms. 517 de la BM de Lyon.

48. Voir par exemple La Cité de Dieu de saint Augustin, Paris, Sainte-Geneviève, ms. 246 .
Le fait est facilement corroboré par l'étude du manuscrit Additional 25695 de la British Library, dont l'illustration est traditionnellement attribuée au Maître de Rolin. Peter Monks y avait déjà isolé une seconde main, sans toutefois l'identifier ${ }^{49}$. Les images de la vie de la Vierge et du Christ sont, à quelques détails près, identiques à celles du Miroir historial de Jacques d'Armagnac et les guides génériques utilisés sont effectivement ceux du Maître de Rolin. Les tours crénelées si particulières, les paysages, les mandorles et les fines architectures dorées qui circonscrivent certaines scènes permettent toutefois d'identifier clairement Maître François comme cette «main $\mathrm{B} »^{50}$

Cette méthode de travail collaborative se poursuit à travers le Miroir historial, probablement jusqu'à la mort du Maître de Rolin. Maître François s'impose progressivement et l'on retrouve dans le fr.51 et le manuscrit 722 de plus en plus de motifs qui lui sont chers et de caractéristiques iconographiques qu'il développe parallèlement dans ses autres travaux. La forme triangulaire des ombres portées, le développement d'un paysage plus riche et varié, l'apparition d'architectures aux colonnes colorées surmontées de fins ornements dorés, l'utilisation répétée des tons violets et l'apparition progressive d'une perspective aérienne sont autant d'indices témoignant de sa responsabilité grandissante dans la mise en image du triple manuscrit de Jacques d'Armagnac. Considérant l'intense activité d'historieur de Maître François, artiste prolifique, il paraît peu probable qu'il ait repris seul l'illustration du Miroir historial après la mort du Maître de Rolin. Il reste alors à déterminer l'identité du troisième enlumineur. L'hypothèse d'une participation du Maître de Jacques de Besançon peut sembler séduisante mais pose toutefois plusieurs problèmes. Le premier est la ressemblance entre les travaux du maître et de l'élève, iconographiquement très proches et donc peu aisés à distinguer. Le second est la date de début d'activité du Maître de Jacques de Besançon, toujours débattue, d'autant plus que la date de fin de la mise en image du Miroir historial n'est pas connue. Il est néanmoins possible d'affirmer que celle-ci se place avant l'arrestation du commanditaire en $1476^{51}$. Sachant que le siège de la demeure de Carlat s'est étendu sur près de dix-huit mois, on peut poser la date de 1475 comme terminus ante quem. Or, si l'on considère que le Maître de Jacques de

49. P. R. Monks, «The Rolin Master's Hand in London BLMS Additional 25695», dan M. M. Manion, B. J.Muir éd., Medieval Texts and Images. Studies of Manuscripts from the Middles Ages, Sydney, 1992, p. 57-70.

50. Maître François, alors au début de son activité, n'a réalisé aucune image complète. Le Maître de Rolin, quant à lui, commençait seulement à s'affranchir de l'influence stylistique du Maître de Bedford.

51. Le nombre d'images étant indiqué à la fin de chacun des trois manuscrits, on peut raisonnablement en déduire qu'ils étaient terminés avant leur dispersion. 
Besançon a commencé son activité au sein de l'atelier vers 1472, il n'a probablement participé qu'à l'illustration du manuscrit 722 .

Cette question n'est que l'un des aspects encore inexplorés - et inexploités - de ce grand miroir. Son examen peut pourtant nous éclairer sur es motivations, personnelles ou politiques, d'un grand commanditaire et son implication dans le processus de création, ou sur l'identification précise des différentes mains qui ont participé à sa décoration et sa mise en image. Une œuvre d'une telle importance, que ce soit dans les moyens déployés autant que dans son aspect bibliophilique général, mériterait une étude approfondie, voire systématique, aussi bien iconographique que stylistique. Si l'immensité de la tâche a de quoi décourager les chercheurs les plus patients, elle permettrait néanmoins de mieux cerner ce prince ambitieux et d'apporter de précieuses informations sur les enlumineurs de l'atelier du Maître de Bedford et, plus généralement, sur l'enluminure du xv siècle.

Cécile RANVIER - Musée de Cluny-Musée national du Moyen Âge

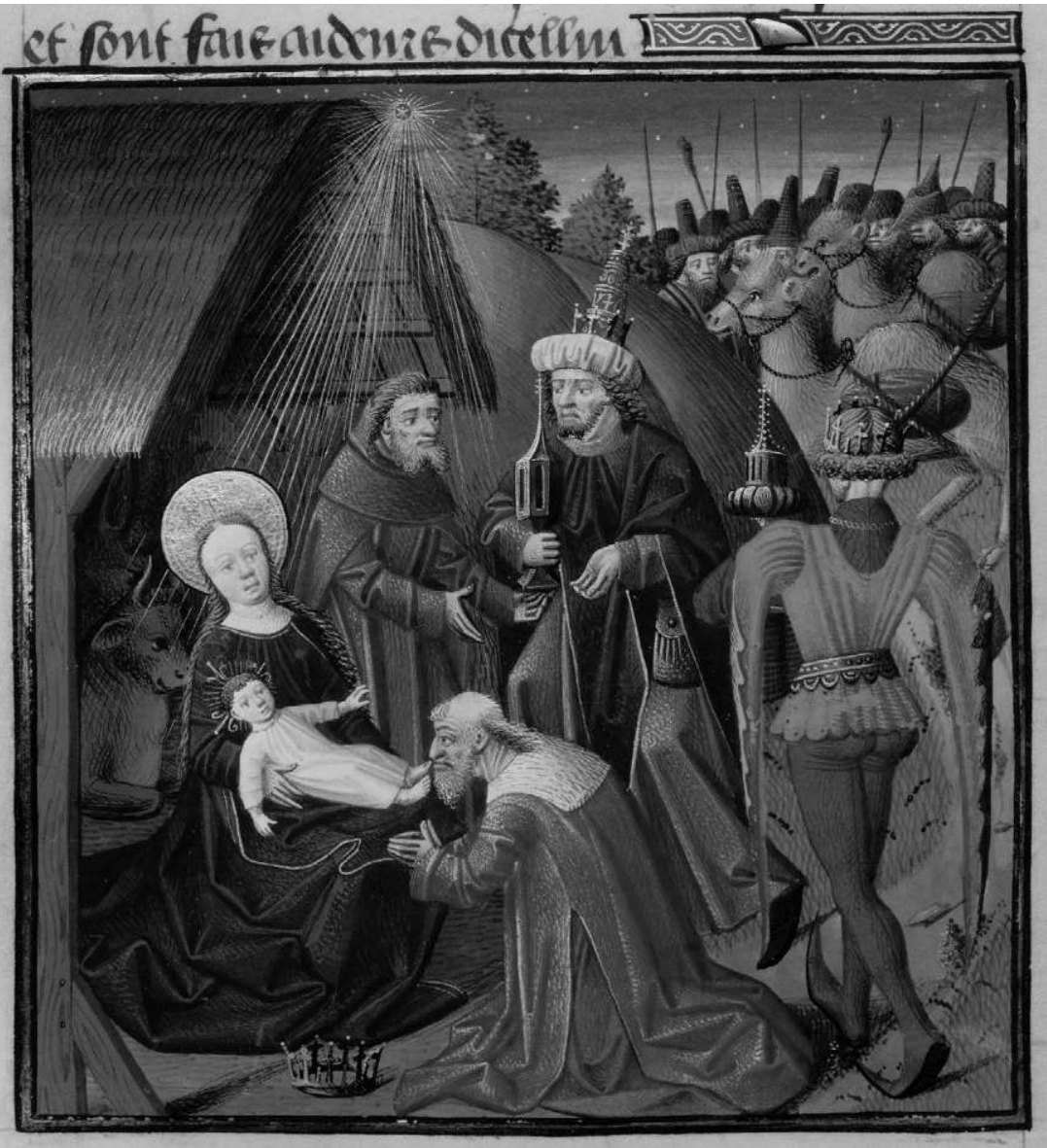

Fig. 1. Miroir historial, Paris, BnF, fr. $50, \mathrm{f}^{\circ} 202 \mathrm{v}^{\circ}$ Maître de Rolin et MaîtreFrançois, L'Adoration des Mages (C BnF). 


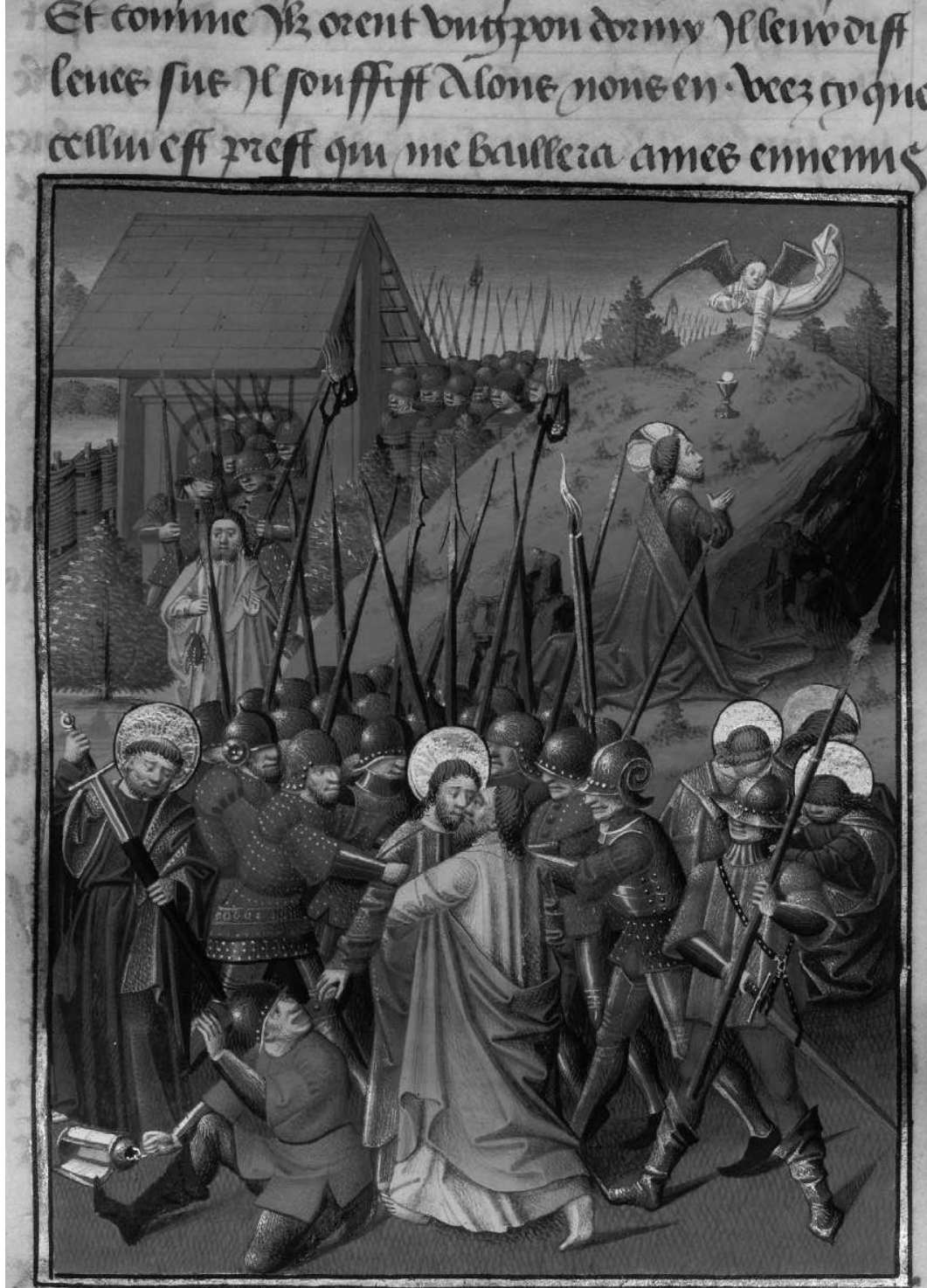

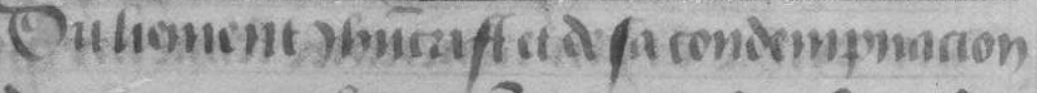

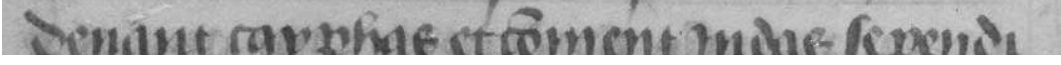

Fig. 2. Miroir historial, Paris, BnF, fr. $50, \mathrm{f}^{\circ} 229 \mathrm{r}^{\circ}$ : Maître de Rolin et Maître François, Le Christ au mont des oliviers, Arrestation du Christ (C) BnF).

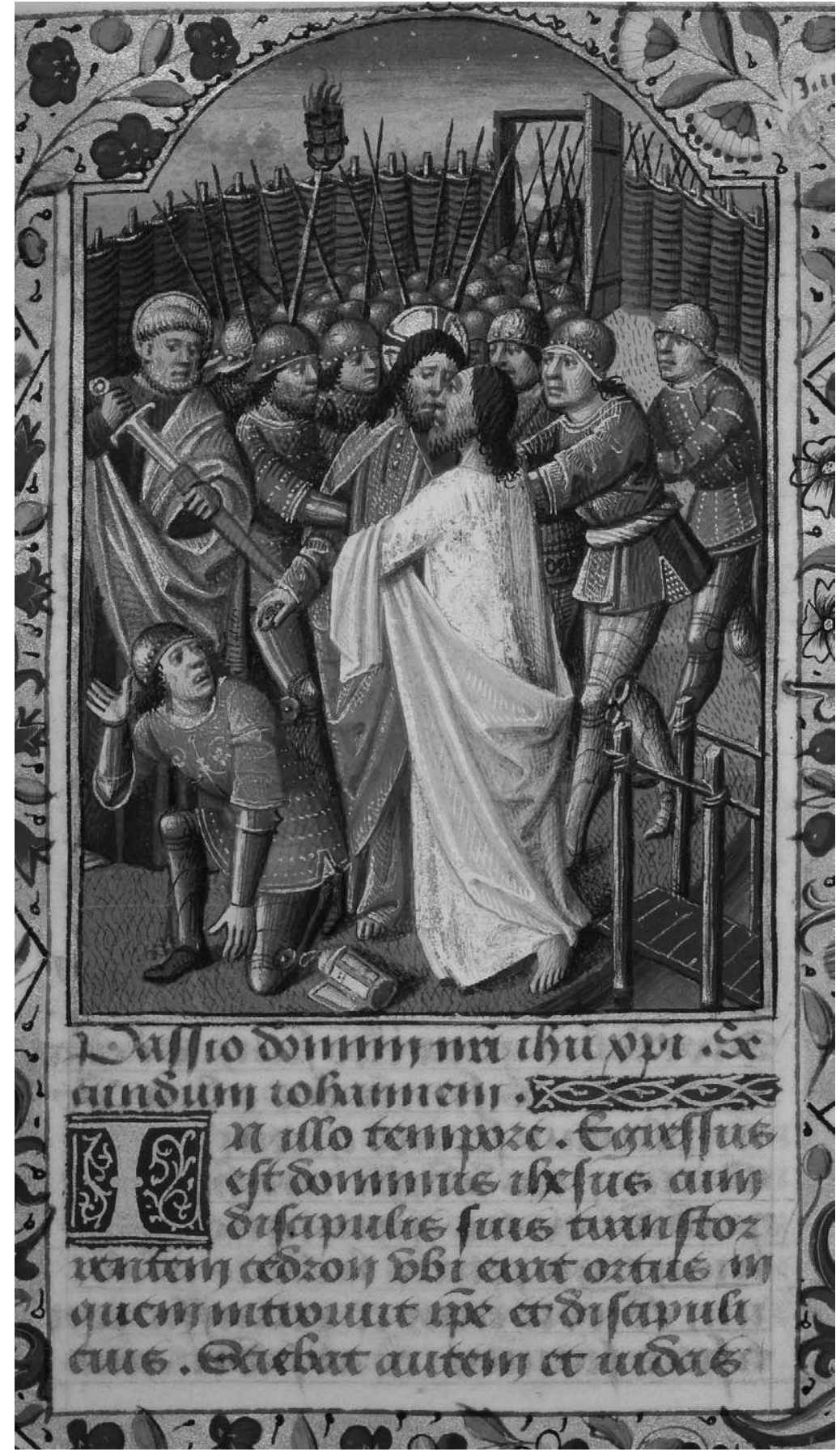

Fig. 3. Livre d'heures de Jacques de Langeac, Lyon, BM, 5154, $\mathrm{f}^{\circ} 113 \mathrm{r}^{\circ}$ : Maître François, Arrestation du Christ (C) BM de Lyon, Didier Nicole). 


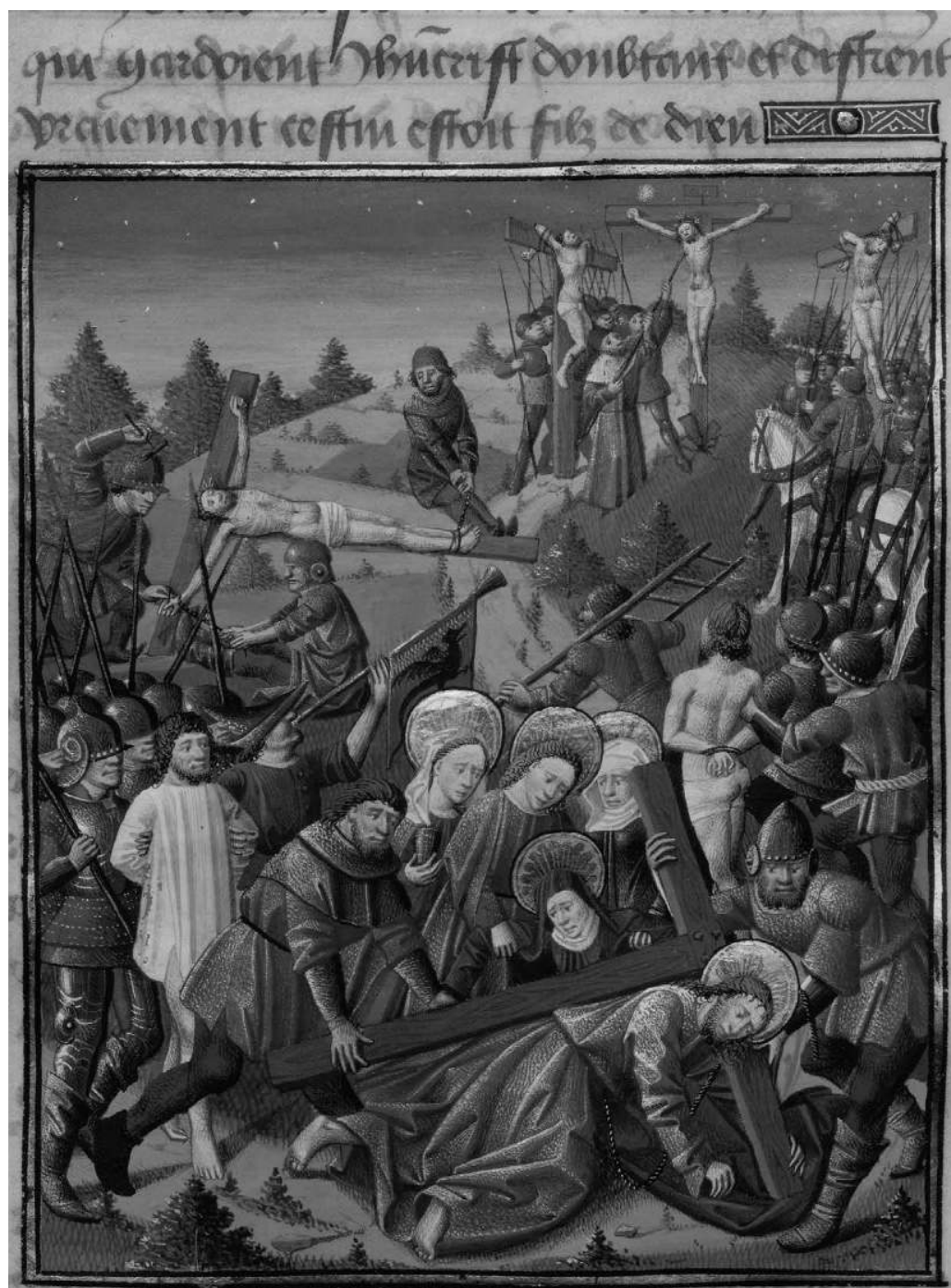

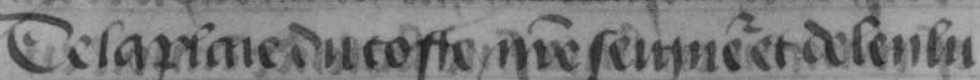

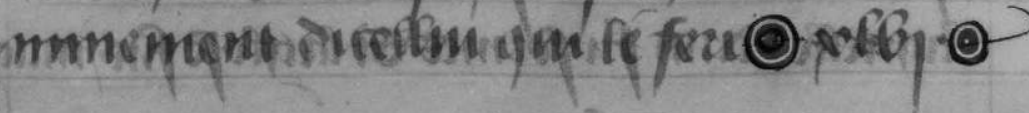

Fig. 4. Miroir historial, Paris, $\mathrm{BnF}$, fr. $50, \mathrm{f}^{\circ} 231 \mathrm{v}^{\circ}$ :

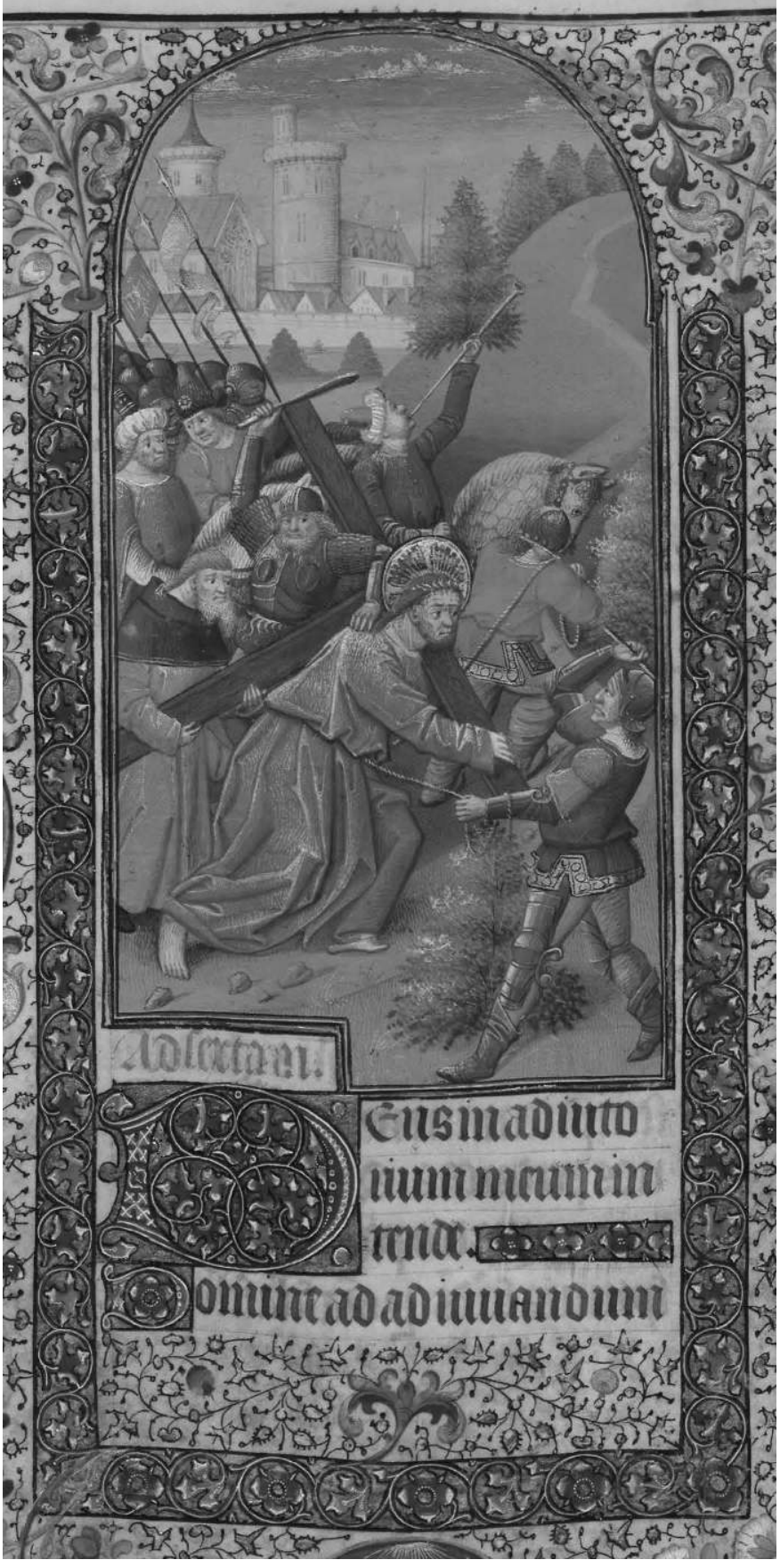

Fig. 5. Livre d'heures, Wien, Österreichische Nationalbibliothek, Codex 1840, $\mathrm{f}^{\circ} 110 \mathrm{v}^{\circ}$ : Maître du duc de Bedford, Portement de Croix (c) Österreichische Nationalbibliothek). 


\section{Le Miroir historial de Jacques d'Armagnac: un monument bibliophilique pour un prince ambitieux}

Lorsque Jacques d'Armagnac commande la première pièce de sa future bibliothèque, un monumental Miroir historial de près de 1350 feuillets, il est alors au sommet de sa carrière politique, soutenu par le roi Louis XI qui a fait de lui l'un des seigneurs les plus puissants de son époque. Plus qu'un simple témoignage de son pouvoir, ce triple manuscrit nous éclaire sur la vie et les aspirations de ce personnage malmené par l'histoire. Le luxe ostentatoire de cet ouvrage se développe à travers les quelque cinq cents miniatures qui l'agrémentent. Réalisées par des enlumineurs particulièrement productifs au cours du xvesiècle, les images du Miroir historial illustrent, par leur relative banalité iconographique, les méthodes de travail des ateliers de l'époque. Elles donnent cependant de précieux indices sur l'identité des différents historieurs qui y ont travaillé et relance, une fois encore, le débat sur l'attribution des images de cet immense manuscrit, un débat qui dure maintenant depuis plus d'un siècle.

cycle marial et christique - Jacques d'Armagnac - Maître de Rolin - Maître François - Miroir historial

The Miroir historial of Jacques d'Armagnac: a Bibliophilic Monument for an Ambitious Prince

When Jacques d'Armagnac ordered the first piece of his coming library - a monumental Miroir historial composed of almost 1350 folios - he is at the summit of his political career, supported by King Louis the XIth who turned him into the most powerful lords of his time. This triple manuscript is far more than the expression of his power; it enlightens us as to the life and the figure, so manhandled by history, he has aspired to be. The luxury of this work is developed through five hundreds miniatures which enliven it. Made during the fifteenth century by particularly productive illuminators, pictures of this Miroir historial illustrate with some kind of banality the working method of the workshops from that time. Nevertheless, it gives important clues on the identity of the illuminators who worked on this great manuscript and reopens again the debate on the attribution of this pictures, an ongoing debate for more than a century.

Jacques d'Armagnac - Maître de Rolin - Marian and Christian cycle - Maître François - Miroir historial 